\title{
Visual texture as a factor in the apparent velocity of objective motion and motion aftereffects
}

\author{
JAMES T. WALKER \\ University of Missouri-St. Louis, St. Louis, Missouri 63121
}

\begin{abstract}
The apparent velocity of an objectively rotating visually textured disk is an increasing monotonic function of the coarseness (size) of visual texture. The apparent velocity of a negative motion aftereffect increases with coarseness of moving induction texture but decreases with coarseness of stationary test texture, and there is an interaction between induction and test textures. An explanation of these effects is based principally on the assumption of greater lateral inhibition between neighboring elements in finer textures.
\end{abstract}

There has been considerable interest in comparisons between real and apparent motion (for example, Kolers, 1963; Scott. Jordan, \& Powell, 1963; Spigel, 1965). Earlier studies have assessed the effects of visual texture on the perceived velocity of either (1) objective motion (Brown, 1931; Oyama, 1970), or (2) motion aftereffects (Cann, 1961; Over, Broerse, Crassini, \& Lovegrove, 1973), but there has been no study directly comparing the effects of visual texture on these two varieties of motion perception. Thus, the present study was undertaken to compare the effects of visual texture on the perceived velocity of objective motion and motion aftereffects.

Visual texture has been shown to influence the apparent velocity of a moving stimulus viewed through an aperture (Brown, 1931; Oyama, 1970). Decreasing the size of a row of dots and concurrently and proportionally decreasing the distance between dots resulted in an increase in apparent velocity (Brown, 1931). When the coarseness of the visual texture was decreased by half, the apparent velocity essentially doubled; Brown referred to this effect as the transposition of velocity. Oyama (1970) found considerably less velocity transposition, but his results agreed in the main with Brown's earlier findings. Other workers (Gibson, 1965; Smith \& Sherlock, 1957) have argued that the greater apparent velocity of Brown's finer textures could be explained on the basis of frequency of appearance and disappearance of texture elements at the edges of the viewing aperture. In order to avoid the possible confounding of apparent velocity with frequency of appearance and disappearance of texture elements, no viewing apertures were used in the present study. The visual textures were presented on rotating disks, and since every texture element was visible at all times,

This research was supported in part by National Institute of Mental Health Grant USPH 1 R03 MH 18809-01. A version of this paper was presented to the Eastern Psychological Association, Washington. D.C.. May 3, 1973. apparent velocity measures were free of any effects of the appearance and disappearance of texture elements. Coarser textures appeared to move faster, and thus the present results run counter to earlier findings (Brown, 1931; Oyama, 1970).

Visual texture also influences motion aftereffects. A rotating spiral produces an aftereffect of expansion or contraction in any subsequently viewed stationary test stimulus, such as a visually textured surface; Cann (1961) found that such aftereffects were greater in fine test textures. The present study used a factorial design to assess the effects of moving induction textures and stationary test textures on the velocity of aftereffects. As in Cann's study, finer test textures produced greater aftereffects. Coarse induction textures produced greater aftereffect velocities, and there was an interaction between induction and test textures.

Recently, texture-contingent visual motion aftereffects have been demonstrated (Mayhew \& Anstis, 1972; Walker, 1972) which appear to be analogous in some respects to the $\mathrm{McC}$ Collough effect (McCollough, 1965 ) and other contingent aftereffects. A coarse visual texture rotating clockwise and a fine texture rotating counterclockwise are presented alternately and are centrally fixated during an induction period of a few minutes. Subsequently, a stationary coarse texture appears to rotate counterclockwise and a stationary fine texture clockwise. Thus, visual texture is an important factor in the perception of motion aftereffects and in the perception of objective motion as well.

\section{EXPERIMENT I}

This experiment was designed to assess the joint effects of objective velocity and visual texture on the apparent velocity of objective motion. In order to facilitate comparisons between objective motion and aftereffect motion, a range of objective velocities was chosen for the present experiment which was expected 
to bracket the velocities of motion aftereffects to be studied in Experinent II.

\section{Method}

Observers. Twelve men and 12 women, introductory psychology students at the University of Missouri-St. Louis, participated as a course requirement.

Apparatus. The visual textures are shown about $3 / 4$ size in Figure 1. This graded series of textures was prepared by making photographic reductions of a master texture consisting of $1 / 4$-in. $(0.4 \mathrm{~mm})$ black dots placed randomly within each $1 / 2-\mathrm{in} .(12.7 \mathrm{~mm})$ square on a large white surface. The coordinates of each dot within its square were determined by choosing a pair of digits from a table of random numbers. Within the limits of the reproduction process, all textures had the same light-dark tatio, namely .80 white and .20 black. neglecting the occasional partial overlap between neighboring texture elements. Various types of visual textures are readily available commercially, but these are mostly ordered arrays of dots or other elements; since Dixon and Meisels (1966) have shown that random arrays produce greater motion aftereffects, random textures were used in the present study. The coarsest (largest) texture in the series is 10 times as coarse as the finest (smallest), and thus the visual textures cover a range of $1.00 \mathrm{log}$ unit. Adjacent textures in the series differ by $.2 \mathrm{log}$ unit. At the viewing distance of $8 \mathrm{ft}(2.4 \mathrm{~m})$, the dots in the coarsest and finest textures respectively subtended visual angles of 8.95 and $.895 \mathrm{~min}$. If the finest texture in the series is assigned a scale value of one on the visual texture dimension, then the scale value of the coarsest is 10. Since the results show that the apparent velocity of objective motion is a power function of visual texture, it is useful to work with the logarithms of scale values, as shown in Figure 1.
Two rotating disks $b$ in. $(15.2 \mathrm{~cm})$ in diam were presented at approximately eye level perpendicular to the observer's line of sight at a viewing distance of $8 \mathrm{ft}(2.4 \mathrm{~m})$. The centers of the disk were 29 in. $(73.3 \mathrm{~cm})$ apart. Each disk subtended a visual angle of $3.58 \mathrm{deg}$, and their centers were separated by $17.37 \mathrm{deg}$. One of the visual textures was mounted on the disk on the right. and a comparison disk, consisting of two black and two white sectors, was mounted on the disk on the left. The disks were rotated by tachometer-feedback-controlled dc motors. A slide projector (100-W tungsten) with a circular aperture in the slide plane illuminated each disk from the front in a sharply defined circle of light. Each projector was powered by a constant-voltage transformer. The disks were housed in boxes so that little light escaped in to the room.

The visual textures and the comparison disk were produced on Eastman PMT photographic paper, which has an essentially matte finish. The space-average luminance of the visual textures. measured with a Macbeth illuminometer, was $40.48 \mathrm{~mL}$ $\left(128.8 \mathrm{~cd} / \mathrm{m}^{2}\right)$ with a standard deviation of $.92 \mathrm{~mL}\left(2.93 \mathrm{~cd} / \mathrm{m}^{2}\right)$. The contrast ratio ${ }^{1}$ was .43 for the visual textures and for the comparison disk.

Procedure. A three-way factorial design was used to assess the effects of sex, objective velocity, and visual texture on the apparent velocity of objective motion. There were repeated measures on the third factor. The six visual textures were presented to each observer in an individually determined random sequence. A texture was placed on the standard disk (always the disk on the right) and rotated at one of three velocities $(.5,2$, or $8 \mathrm{rpm})$. The observer adjusted the velocity of the comparison disk (always on the left) by turning a knob until its apparent velocity matched that of the standard. The standard and comparison were both lighted, and the observer was instructed to look at the center of each disk while
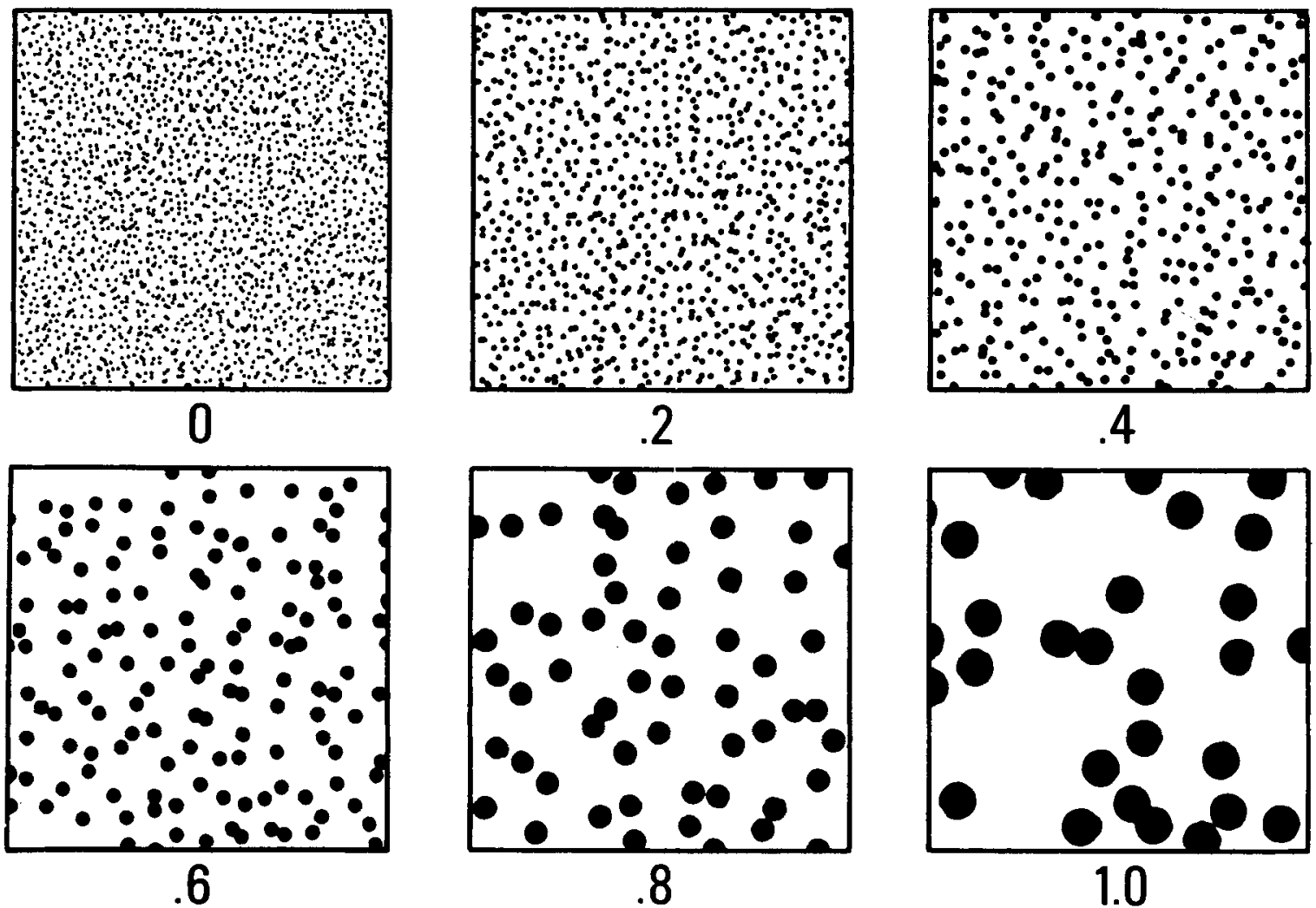

Figure 1. Visual textures (about $3 / 4$ size) ranging from fine through coarse, 0 through 1.0 in $\log$ units. 
making adjustments. Most observers required less than $30 \mathrm{sec}$ to make a satisfactory velocity match on each trial. The velocity of the comparison disk was recorded automatically on a chart recorder. On any given trial, both disks rotated in the same direction, but on successive trials. clockwise and counterclockwise rotations were alternated to help control the buildup of motion aftereffects. There was a period of about $30 \mathrm{sec}$ between successive trials, sufficient for the experimenter to change the texture on the standard disk. When all six visual textures had been presented to an observer, the textures were presented once again in reversed sequence; thus, the orders of textures were counterbalanced within observers and randomized between observers.

\section{Results}

The data were transformed logarithmically, and an analysis of variance was carried out. Figure $2 \mathrm{~A}$ shows the results graphically. The effect of objective velocity was highly signiticant. $F(2,18)=471.76, p<.001$, and there was a significant texture effect, $F(5.90)=$ $10.67, p<.01$. There was no significant sex effect, $\mathrm{F}(1,18)=4.15, \mathrm{p}<.10$, and no interaction approached significance. The least squares regression lines in Figure 2A show that apparent velocity of objective rotation increases with increasing coarseness of visual texture at all three levels of objective velocity. The regression equation used to plot the above regression lines is the following:

$$
\log \mathrm{V}_{\mathrm{a}}=-.0052+.94 \log \mathrm{V}_{\mathrm{o}}+.099 \log \mathrm{T}
$$

where $\mathrm{V}_{\mathrm{a}}=$ apparent velocity, $\mathrm{V}_{\mathrm{o}}=$ objective velocity, and $T=$ coarseness of visual texture. Thus, apparent velocity is a power function of objective velocity, where the exponent is .94 , and visual texture, where the exponent is .099 . Although objective velocity is a much greater factor than visual texture in the above equation, the range of textures used in the present experiment produced a change of $.099 \mathrm{log}$ unit $(25.6 \%)$ in the apparent velocity of the rotating disks. The effects of texture are statistically significant, and also consistent across the levels of objective velocity, as shown by the absence of any interaction.

\section{EXPERIMENT II}

The present experiment was designed to assess the effects of moving induction textures and stationary test textures on the apparent velocity of visual motion aftereffects.

\section{Method}

Observers. Twelve men and 12 women, introductory psychology students. participated as a course requirement. One potential observer was excluded, as set out below.

Apparatus. The same apparatus was used as in Experiment I. Only three visual textures were used in the present experiment: 0 . .4 , and $.8 \log$ unit.

Procedure. A three-way factorial design was used in the present experiment. The factors were sex, induction texture, and test texture. There were repeated measures on the last two factors. Each
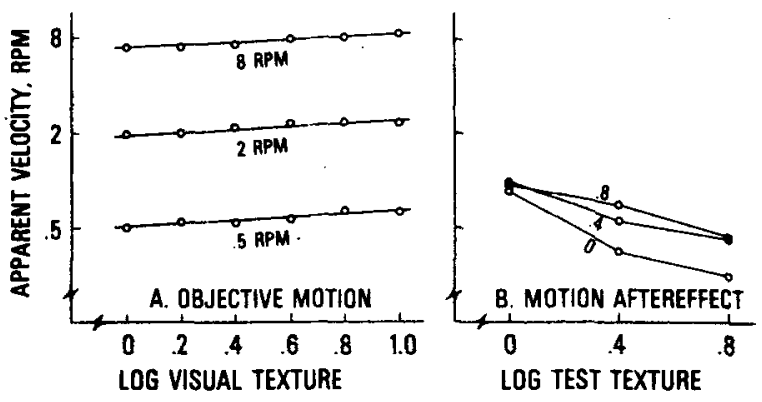

Figure 2. (A) Apparent velocity of objective motion as a function of visual texture. Parameter is objective rate of rotation. (B) Apparent velocity of motion aftereffect as a function of test texture. Parameter here is texture of induction stimulus (rotating disk).

observer was presented all nine combinations of three induction and three test textures in an individually determined random order. The induction texture was always placed on the right disk and was always rotated clockwise at $8 \mathrm{rpm}$. After a short dark-adaptation period, each observer was given two practice trials. The observer was asked to fixate the center of a rotating induction texture $(.8 \mathrm{log}$ unit), and after $15 \mathrm{sec}$ of fixation, the observer was shown a stationary test texture $(0 \mathrm{log}$ unit) on the comparison disk. No observer failed to see a negative motion aftereffect at this point. The observer was then shown how to adjust the test disk by turning a knob so that objective clockwise rotation of the test disk would cancel the apparent motion of the counterclockwise negative motion aftereffect. Another practice trial was given before continuing. One potential observer was replaced because of an inability to follow instructions. In the experiment proper, a small red warning light came on $5 \mathrm{sec}$ before the induction period. During the 30 -sec induction period, only the induction disk was lighted. At the end of the induction period, the induction disk was darkened and the test was lighted. The observer was instructed to fixate the center of the test disk and adjust its velocity so that the disk appeared stationary. The aftereffect velocity was measured by automatically recording the objective velocity at which the test disk appeared stationary. At the end of the 15 -sec test period, the test disk was darkened. The intertrial interval between the end of a test period and the beginning of an induction period was $60 \mathrm{sec}$, and the observer remained in darkness during this period. The presentation of stimuli and the timing of intertrial intervals were all controlled by Hunter timers.

\section{Results}

For each 15-sec test trial, each observer's average velocity was read from the chart recorder. The data were transformed logarithmically before carrying out an analysis of variance; since some of the aftereffect measures were equal to zero, a $\log (X+1)$ transformation was used. Figure $2 B$ shows the results graphically. The effect of test texture was highly significant, $F(2,44)=86.25, \mathrm{p}<.001$; so was the effect of induction texture, $F(2,44)=18.71$, $\mathrm{p}<.001$; and there was a significant Induction by Test Texture interaction, $F(4,88)=2.53, \mathrm{p}<.05$. The simple effects of test texture were highly significant at all three levels of induction texture, all Fs $(2.132)>23.00, p<.001$. There was no simple effect of induction texture at the level of the finest test texture. $F(2,132)=1.39, p>.25$, and thus, all three induction textures produced essentially equal-and 
strong-aftereffects on the finest test texture. Finally, there was no sex effect, $F(1.22)=1.11, p>.25$.

\section{DISCUSSION}

In Experiment I, objectively moving coarse textures produced greater apparent velocities than fine textures. Differing amounts of lateral inhibition between neighboring elements in fine and coarse textures may provide a possible explanation for the relationship between visual texture and apparent velocity. The hypothetical distributions of neural activity generated by coarse and fine textures are illustrated in Figures $3 \mathrm{~A}$ and $3 \mathrm{~B}$, respectively, where each texture element generates a narrow area of neural excitation and a wider area of neural inhibition (Békésy, 1967). For moving stimuli, the distribution of excitation is probably asymmetrical, as Spitz (1958) has argued, but any such asymmetry can be neglected in the present discussion, which will assume the same distribution of excitation and inhibition in moving as in stationary textures. In a coarse texture, there is little mutual inhibition between elements because of their spacing, but in a fine texture, there is considerable mutual inhibition between neighboring elements. Thus, in a fine texture, the peak of neural excitation generated by each element is lower, as show $n$ by the dotted lines in Figure 3B. If the greater excitation associated with the elements of a coarse texture is a more effective stimulus for motion detectors than is the lower excitation associated with a fine texture, then a coarse texture might appear to move faster than a fine texture moving at the same objective velocity.

The results of Experiment II are consistent with the earlier study of Cann (1961), who found that a rotating spiral produced greater aftereffects in fine-textured stationary test stimuli. In the present study, induction as well as test textures were

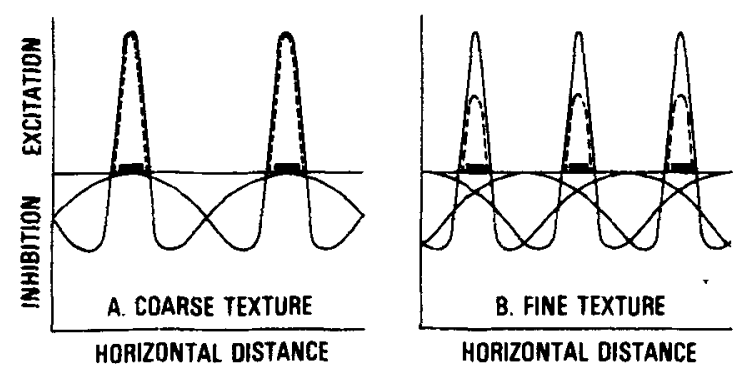

Figure 3. Distributions of neural activity for a coarse texture (A) and a fine texture $(B)$. Solid bars indicate locations of texture elements. Solid lines indicate the distributions of excitation and inhibition which each texture element would generate by itself, in the absence of lateral inhibition from neighboring texture elements. Dashed lines indicate reduced excitation due to lateral inhibition. Peaks of excitation are lower in fine texture (B) due to greater mutual inhibition. manipulated, and aftereffect velocity was shown to increase with coarseness of induction texture and to decrease with coarseness of test texture. However, in a recent study of motion aftereffects produced by moving gratings (Over et al., 1973), coarser stationary test gratings produced greater aftereffect velocities, contrary to the present results and to Cann's as well. Although gratings are very different from the stimuli used in the present study, it may be useful to compare the coarseness of the textures used here with the coarseness of the gratings used in the Over et al. study, where the gratings ranged from $7.5 \mathrm{~min}$ to 2 deg of visual angle per cycle. In the present textures, if each unit area containing a dot is considered as a "cycle," then the present textures range from .895 to 8.95 min of visual angle per cycle. Thus, the coarsest of the present textures corresponds roughly with the finest grating in the Over et al. study. [In order to construct a random-dot texture roughly corresponding with the coarsest grating in the Over et al. study, the dots would need to be about $3.5 \mathrm{in} .(8.9 \mathrm{~cm})$ in diam and would be located in unit squares about 7 in. $(17.8 \mathrm{~cm})$ on a side.] The following observations suggest a possible explanation for the finding that coarse test gratings produced greater aftereffects in the Over et al. study. Motion aftereffects can be seen on extremely fine visual textures, such as a blank sheet of paper, and can even be seen with the eyes closed, where the visual texture can be considered infinitely fine; indeed, aftereffect velocities may be very high under those conditions (Holland, 1965). An extremely coarse visual texture-where a single element would fill the field of view-would reduce in effect to a blank sheet of paper, and thus might produce a great aftereffect velocity if it were presented as a test texture. Thus, the relationship between aftereffect velocity and test texture may be a $U$ function across the full range of possible visual textures. In the Over et al. study, each black and white stripe in the coarsest grating may have been large enough to reduce in effect to a blank area of infinitely fine texture, which could be expected to produce a great aftereffect velocity.

The results of Experiment I, which showed that visual texture was a factor in the apparent velocity of objective motion, suggest a possible explanation for the effects of induction textures on the apparent velocity of the visual motion aftereffects. In the present experiment, the induction textures always rotated at $8 \mathrm{rpm}$. If this speed of rotation were below the optimum speed for the production of a motion aftereffect, then an increased speed of rotation would produce a stronger motion aftereffect. Since objectively rotating coarser textures appear to move faster, it might follow that coarser textures would therefore produce stronger motion aftereffects. Such an explanation, however, appears somewhat unlikely in the light of earlier studies. Dixon and Meisels 
(1966) found that the magnitude of motion aftereffects produced by a rotating disk reached a maximum at $2 \mathrm{rpm}$ and then declined monotonically through their maximum velocity of $6 \mathrm{rpm}$, and Sekuler and Pantle (1967) found that aftereffect magnitude decreased monotonically from 2.5 through $20.5 \mathrm{rpm} .{ }^{2}$ While the stimuli in those earlier studies were very different from the present stimuli, those earlier results suggest that a higher speed of rotation in the present experiment should produce a smaller rather than a greater motion aftereffect.

An alternative explanation better accounts for the effects of induction texture on aftereffect velocity. In Experiment I, objectively moving coarse textures appeared to move faster than fine textures, and thus coarse textures were shown to be more effective stimuli for motion perception, insofar as apparent velocity is concerned. Coarse induction textures may produce stronger motion aftereffects because those textures are more effective in stimulating and fatiguing motion detectors.

The effect of test texture on the motion aftereffect is more difficult to explain. Cann (1961) has argued that the texture elements in finer textures are subject to more mutual inhibition than those in coarser textures, and that finer test textures are therefore less "stable," and thus produce greater motion aftereffects. But Cann offers no explanation for the direction of negative motion aftereffects, and without additional assumptions, it is difficult to see how the "instability" of finer textures could lead to greater motion aftereffects.

Several workers have argued (for example, Sekuler \&Pantle, 1967) that a negative motion aftereffect results from the differential adaptation and fatigue of different sets of motion detectors during the presentation of a moving stimulus. If it is assumed that a subsequently presented stationary test stimulus also stimulates motion detectors, then the more fatigued motion detectors would respond at a lower rate than the unadapted motion detectors. The difference between the response rates in these two sets of motion detectors would give rise to a negative motion aftereffect. Experiment I showed that coarse textures are more effective than fine textures as stimuli for objective motion perception, and presumably more effective stimuli for motion detectors. If it is assumed that stationary coarse textures also stimulate motion detectors more effectively, then the following explanation becomes possible. A fine stationary test texture, which is assumed to be a weak stimulus for motion detectors, may not stimulate any activity in the set of motion detectors previously adapted to motion in a particular direction. But the unadapted detectors may respond to the fine test texture, and the difference in response rates between the adapted and unadapted motion detectors may result in a negative motion aftereffect. A stationary coarse test texture, which is assumed to be a stronger stimulus for motion detectors, may stimulate a substantial level of activity in the fatigued as well as the unadapted motion detectors, and in that case there would be little motion aftereffect. It is necessary to assume that a moving texture stimulates motion detectors more than a stationary one, otherwise there could be no perception of objective motion, since a coarse texture-moving or not-would equally stimulate all motion detectors for all directions of motion. These assumptions are consistent with the findings of Hubel and Wiesel (1962). Some complex cells in the visual cortex of the cat were found to respond at substantial rates to properly oriented stationary bars of light, but the highest sustained rates of response were produced by bars of light moving in a preferred direction. Thus, if those complex cells are considered motion detectors, then it is clear that such motion detectors respond to stationary as well as moving stimuli.

The present explanations for the effects of visual texture on motion aftereffects were largely derived from explanations offered earlier by Keck, Palella, Carroll, and Pantle (Note 1) for the effects of luminance contrast on motion aftereffects. Keck et al. found that the apparent velocity of motion aftereffects increased with increasing contrast of moving induction gratings but decreased with increasing contrast of stationary test gratings; those workers argued that a stationary test stimulus of high contrast stimulates both the adapted and unadapted sets of motion detectors at nearly the same rate, thus producing only a slight motion aftereffect, while a test stimulus of lower contrast stimulates the two sets of motion detectors less strongly, thus permitting a substantially lower rate of activity in the adapted motion detectors. If the coarseness of visual textures and the luminance contrast of visual stimuli play functionally similar roles in stimulating motion detectors, then the explanation which Keck et al. offered for the role of luminance contrast in determining aftereffect magnitude can be extended to account for the role of visual texture as a factor in aftereffect magnitude. It is also possible that brightness contrast may play some role in the present study: The visual textures used here were essentially unidimensional, within the limits of the photographic process, in that the light-dark ratio, the objective luminance contrast, and the space-average luminance were all essentially constant. But to most observers, the finer visual textures appear darker and also appear to have lower contrast than the coarser textures. While subjective brightness and subjective contrast were not measured in the present study, it 
may be that these dimensions play some role in the apparent velocity of motion aftereffects and objective motion as well.

The assumption of greater lateral inhibition between the elements of finer textures helps to account for the lower apparent velocity of objectively moving tine textures and also for the higher apparent velocity of motion aftereffects seen in fine stationary test textures. That assumption also helps to account for the higher aftereffect velocities produced by objectively moving coarse induction textures. However, the proposed explanations for the relationships between apparent velocity and visual texture are highly speculative in the absence of any direct supporting neurophysiological evidence.

\section{REFERENCE NOTE}

1. Keck. M. J., Pallela, T. D., Carroll, J., \& Pantle, A. Motion aftereffect as a function of the contrast of sinusoidal gratings. Paper presented to the Association for Research in Vision and Ophthalmology, Sarasota. Florida, May 1973.

\section{REFERENCES}

BÉKÉsy. G. yoN. Sensory inhibition. Princeton, N.J: Princeton University Press. 1967.

Brown. J. F. The visual perception of velocity. Psychologische Forschung, 1931, 14, 199-232.

CANN. M. A. The negative after-effect of motion as a function of test stimulus texture. (Doctoral dissertation. Boston University.) Ann Arbor. Michigan: University Microfilms, 1961 , No. 61-2414.

Dixon. N. F., \& Meisels, L. The effect of information content upon the perception and after-effects of a rotating field. Quarterly Journal of Experimental Psychology, 1966, 18. 310-318.

Gibson. J. J. Research on visual perception of motion and change. Second Symposium on Physiological Psychology. School of Aviation Medicine, Pensacola. Florida. 1958. In I. M. Spigel (Ed.). Readings in the study of visually perceived movement. New York: Harper \& Row, 1965. Pp. 125-146.

Holland, H. C. The spiral after-effect. Oxford: Pergamon Press. 1965. Hubel, D. H., \& WIESEL, T. N. Receptive fields. binocular interaction, and functional architecture in the cat's visual cortex. Journal of Physiology, 1962, 160, 106-154.

Kolers. P. A. Some differences between real and apparent visual movement. Vision Research, 1963, 3, 191-206.

MaYhew. J. E. W., \& Anstis, S. M. Movement aftereffects contingent on color, intensity, and pattern. Perception \& Psychophysics, 1972. 12. 77-85.

McCollough. C. Color adaptation of edge-detectors in the human visual system. Science. 1965, 149. 1115-1116.

Over, R., Broerse. J., Crassini, B., \& Lovegrove, W. Spatial determinants of the aftereffect of seen motion. Vision Research. 1973, 13. 1681-1690.

Oy ama. T. The visually perceived velocity as a function of aperture size, stripe size. luminance, and motion direction. Japanese Psychological Research. 1970, 12. 163-171.

Scotr, T. R., Jordan, A. E., \& Powell, D. A. Does visual aftereffect of motion add algebraically to objective motion of the test stimulus? Joumal of Experimental Psychology, 1963, 66. 500-505.

Sekuler, R., \& Pantle, A. A model for after-effects of seen movement. Vision Research, 1967, 7, 427-439.

Smith, O. W.. \& Sherlock, C. A. A new explanation of the velocity transposition phenomenon. American Journal of Psychology, 1957. 70. 102-105.

SpIGEL, I. M. (Ed.) Readings in the study of visually perceived movement. New York: Harper \& Row, 1965.

SpITZ, H. H. Neural satiation in the spiral aftereffect and similar movement aftereffects. Perceptual and Motor Skills, 1958, 8. $207-213$.

WALKER, J. T. A texture-contingent visual motion aftereffect. Psychonomic Science, 1972, 28, 333-335.

\section{NOTES}

1. The contrast ratio is defined by the following formula: $\mathrm{C}=\left(\mathrm{L}_{\max }-\mathrm{L}_{\min }\right) /\left(\mathrm{L}_{\max }+\mathrm{L}_{\min }\right)$, where $\mathrm{C}=$ contrast ratio, $\mathrm{L}_{\max }=$ maximum luminance, and $\mathrm{L}_{\min }=$ minimum luminance.

2 . It is of course true that the linear velocity of any point in the retinal image of a centrally tixated rotating disk is determined not only by the rate of rotation, but also by the radial distance of the point from the center of rotation. It is difficult to make comparisons across different experiments, since the linear velocities of points on the edges of rotating disks vary with disk size, viewing distance, and rotation rate. But consider two disks which subtend different visual angles. and thus project different sizes of retinal images. Both disks rotate at the same rate, but the linear velocity of a point on the edge of the disk subtending the larger visual angle is greater than the velocity of a point on the edge of the other disk. However. there is a central region within the larger retinal image-swept out by a radius equal to that of the smaller retinal image of the other disk-where the linear velocity of every point is equal to that of every corresponding point in the smaller retinal image of the disk subtending the smaller visual angle. Thus, rotation rates may provide meaningful comparisons across different sizes of disks and different viewing distances.

(Received for publication August 23, 1974; revision received May 2, 1975.) 